\title{
EDITORIAL
}

\section{Minimally invasive surgery in urogynecology}

\author{
Peter L. Dwyer
}

Received: 12 February 2009 / Accepted: 21 February 2009/Published online: 20 March 2009

(C) The International Urogynecological Association 2009

Minimally invasive surgery has been a term used to describe laparoscopic surgery and more recently the trocar-guided synthetic mid-urethral slings for stress incontinence and the trocar-guided synthetic mesh kits to treat prolapse. This term implies a lesser operation, more pain free and safer, which in many cases is not true. This description seems more to do with marketing the product and the surgeon than actually describing the benefits and risks of the procedure. When complications occur as they always will, they may be more unexpected by the patient and poorly tolerated. Therefore informed consent, giving the patient a clear understating of the procedure, possible risks and benefits, and likely outcome, is essential.

Synthetic polypropylene slings (SPS) are not new and were used to treat women with stress incontinence by Morgan in Northern America and Moir in the UK in the 1960s. These slings were placed under the bladder neck through open abdominal and vaginal incisions. However, it was not until the mid-1990s with the introduction of the tension-free vaginal tape (TVT) that SPS gained widespread usage when placed through small incisions using trocar needles at the mid-urethral position. These retropubic slings have been found to have benefits of shorter hospitalization, less postoperative pain and a lower morbidity than the Burch colposuspension and fascial slings but equal effectiveness. Over the last 6 years, numerous variations of the TVT have been introduced, including the transobturator approach and the no-incision mini-slings (TVT Secur, Gynecare Ethicon Inc., Somerville, NJ) and Miniarc (American Medical Systems Inc., Minnetonka,

P. L. Dwyer $(\bowtie)$

Mercy Hospital for Women,

Melbourne, Australia

e-mail: pdwyer@connexus.net.au
MN). A major advantage of the transobturator and minislings is the avoidance of the retropubic space, decreasing complications such as bladder and bowel perforation, and major vascular injury. Bladder perforation in retropubic slings is a common complication with reported rates of $0.7 \%$ to $34.2 \%$ compared to $0 \%$ to $3.1 \%$ in the transobturator approach [1]; if recognized, sling replacement is performed without clinical sequel. Bowel and vascular injuries are more serious and have resulted in patient deaths. These complications have resulted in many surgeons changing from retropubic slings or preferring to learn the other approaches. Current sales of these stress incontinence devices according to industry sources are "obturator slings make up just over half of the total synthetic sling procedures performed in the US. Newer single incision slings represent between $15 \%-20 \%$. The remaining $30 \%$ are retropubic slings. This is across all major/medium size brands."

However, is this change away from retropubic slings for perceived safety reasons premature and in the patient's best long-term interests? Prospective randomized studies [1] comparing the retropubic and transobturator slings in woman with non-intrinsic sphincter deficiency (non-ISD) stress urinary incontinence (SUI) have shown no significant difference in effectiveness, although follow-up in these studies is of short duration (less than a year) and based on symptoms only. However, short-term subjective outcomes may not be indicative of more long-term results. In a study comparing pubovaginal sling to transurethral Macroplastique injection in ISD patients [2] the subjective symptomatic improvement was not significantly different at 6 months, but urodynamic SUI cure at 6 months, $(81 \%$ vs. $9 \%)$ and 5 year symptomatic cure was significantly in favor of the pubovaginal fascial sling ( $69 \%$ vs. $21 \%$ ). In women with ISD, SUI the retropubic TVT sling has been shown to be more 
effective than the transobturator Monarc sling [3]. There is little evidence to date of the effectiveness of the no-incision mini-slings. Therefore, long-term studies with objective assessment are needed to determine the relative efficacy of all mid-urethral sling procedures before the extensively investigated TVT retropubic sling should be replaced as the gold standard.

Perhaps in part propelled by the success of the synthetic mid-urethral slings, trocar-placed polypropylene graft kits are increasing being used for women with pelvic organ prolapse (POP). There are a number of retrospective studies confirming the short-term effectiveness of these devices but the complications are of concern. A recent retrospective multi-center study [4] of "the new minimally invasive mesh repair devices" of 329 women reported operative complications which included bladder injury (1.6\%), rectal injury $(1.1 \%)$ and two women with serious vascular injuries. Other postoperative complications included: buttock pain $(5.2 \%)$, vaginal erosion $(10 \%)$, one woman with bladder erosion, and two women $(0.7 \%)$ with serious infection, leading to necrotizing fasciitis in one woman. Injury to pelvic organs can occur with fascial non-mesh repairs but the mesh erosion, vascular injuries, and buttock pain are related to these devices and their placement. It would seem appropriate at this stage until there is conclusive evidence that these devices are used in recurrent large vaginal prolapse by experienced surgeons and not seen as a simple quick fix solution to all POP.

Complications occur with all surgery. The US Food and Drug Administration (FDA) has recently taken the unusual step of warning the public of complications that can occur when surgical mesh is used to treat POP and SUI, and advised patients to discuss fully these risks with doctors prior to surgery and report to them any adverse events postoperatively.

Finally, when complications occur, they need to be dealt with effectively and safely, sometimes with the aid of a urologist or colorectal surgeon. Recently, I have seen a simple vaginal mesh erosion become a rectovaginal fistula following excision surgery, and have had two women with urethrovaginal fistula referred following revision surgery for voiding difficulty after transobturator slings. The insertion of the devices may be relatively straightforward but the management of mesh complications when they occur should be by very experienced surgeons.

\section{References}

1. Latthe PM, Foon R, Toozs-Hobson P (2007) Transobturator and retropubic tape procedures in stress urinary incontinence: a systematic review and meta-analysis of effectiveness and complications. BJOG 114:522

2. Maher C, O'Reilly BA, Dwyer PL, Carey M, Cornish A, Schluter P (2005) Pubovaginal sling or transurethral Macroplastique for stress urinary incontinence and intrinsic sphincter deficiency: a prospective randomized trial. BJOG 112:797-801

3. Schierlitz L, Dwyer PL, Rosamilia A, Murray C, Thomas E, De Souza A, Lim Y, Hiscock R (2008) Tension free vaginal tape is more effective than the trans-obturator tape in women with stress urinary incontinence and intrinsic sphincter deficiency. A prospective randomized study. Obstet Gynecol 112(6):1253-1261

4. Abdel-Fattah M, Ramsay I (2008) Retrospective multicentre study of the new minimally invasive mesh repair devices for pelvic organ prolapse. BJOG 115(1)):22-30 